\title{
Qualidade do sono e sua relação com qualidade de vida e estado emocional em professores universitários
}

\author{
Quality of sleep and its relationship to quality of life and \\ emotional status in university professors
}

\section{Calidad del sueño y su relación con calidad de vida y estado emocional en profesores universitarios}

\author{
Maurilo Aparecido Borges ${ }^{1}$, Débora Almeida Galdino Alves ${ }^{2}$, \\ Laiz Helena de Castro Toledo Guimarães ${ }^{3}$
}

\begin{abstract}
1. Acadêmico do Curso de Graduação em fisioterapia do Centro Univeristário de Lavras (Unilavras). LavrasMG, Brasil.

2.Fisioterapeuta, Doutora e Docente do Curso de Fisioterapia do Centro Univeristário de Lavras (Unilavras). Lavras-MG, Brasil.

3.Fisioterapeuta, Mestre e Docente do Curso de Fisioterapia do Centro Univeristário de Lavras (Unilavras). Lavras-MG, Brasil.
\end{abstract}

\begin{abstract}
Resumo
Introdução. Compreender o padrão de sono em professores universitários mostra-se necessário, uma vez que há consequências físicas, mentais e ocupacionais negativas que podem interferir, entre outros aspectos, na qualidade de vida e estado emocional. Objetivo. Avaliar a qualidade do sono e sua relação com a qualidade de vida e estado emocional em professores universitários. Método. Trata-se de um estudo transversal, no qual participaram 36 professores de uma instituição de ensino superior privada no interior do estado de Minas Gerais, sendo $27,8 \%$ do gênero masculino e $72,2 \%$ do gênero feminino, com idade média entre 25 e 61 anos. Instrumentos utilizados: questionário sociodemográfico, Índice de Qualidade do Sono de Pittsburgh (IQSP), questionário de qualidade de vida SF-36 e Escala de ansiedade, depressão e estresse (EADS-21). Resultados. 58,3\% dos participantes apresentaram qualidade do sono ruim. Professores com pior qualidade do sono, apresentaram pior qualidade de vida $(p<0,05)$ e mais sintomas de ansiedade $(p=0,00078)$. Conclusão. Pode-se concluir que a má qualidade do sono é um problema comum entre os professores analisados e está associada a baixa qualidade de vida e ansiedade.
\end{abstract}

Unitermos. Sono; Professores universitários; Qualidade de vida; Aspectos emocionais

\begin{abstract}
Introduction. Understanding sleep patterns in university professors is necessary, since there are negative physical, mental, and occupational consequences that may interfere, among other things, with quality of life and emotional state. Objectives. Assess the quality of sleep and its relationship to quality of life and emotional state in university professors. Method. This is a cross-sectional study, in which 36 professors participated in the of a private higher education institution in the state of Minas Gerais, $27.8 \%$ male and $72.2 \%$ female, with average age between 25 and 61 years. Instruments used: sociodemographic questionnaire, Pittsburgh Sleep Quality Index (IQSP), SF-36 quality of life questionnaire and Anxiety, depression, and stress scale (EADS-21). Result. 58.3\% of the participants presented poor sleep quality. Teachers with worse sleep quality had worse quality of life $(P<0.05)$ and more anxiety symptoms $(p=0.00078)$. Conclusion. It can be concluded that poor quality of sleep is a common problem among the teachers analysed and is associated with low quality of life and anxiety.
\end{abstract}

Keywords. Sleep; professors; Quality of life; Emotional aspects 


\section{Resumen}

Introducción. Comprender el patrón de sueño en los profesores universitarios resulta necesario, ya que hay consecuencias físicas, mentales y ocupacionales negativas que pueden interferir, entre otros aspectos, en la calidad de vida y el estado emocional. Objetivo. Evaluar la calidad del sueño y su relación con la calidad de vida y el estado emocional en los profesores universitarios. Método. Se trata de un estudio transversal, en el cual participaron 36 profesores de una institución de educación superior privada en el interior del estado de Minas Gerais, siendo $27,8 \%$ del género masculino y $72,2 \%$ del género femenino, con edad media entre 25 y 61 años. Instrumentos utilizados: cuestionario sociodemográfico, Índice de calidad del sueño de Pittsburgh (IQSP), cuestionario de calidad de vida SF-36 y escala de ansiedad, depresión u estrés (EADS-21). Resultado. El 58,3\% de los participantes tenía mala calidad del sueño. Los profesores con peor calidad del sueño tenían peor calidad de vida $(p<0,05)$ y más síntomas de ansiedad $(p<0,001)$. Conclusión. Se puede concluir que la mala calidad del sueño es un problema común entre los docentes analizados y se asocia con baja calidad de vida y ansiedad.

Palabras clave. Dormir; Profesores universitarios; Calidad de vida; Aspectos emocionales

Trabalho realizado no Centro Univeristário de Lavras (Unilavras). Lavras-MG, Brasil.

\section{INTRODUÇÃO}

Alterações no estilo de vida da sociedade, conduziu a um aumento progressivo na duração da vigília e uma negligência sobre a importância do sono ${ }^{1}$, como agente reconstrutor. Essas alterações tem modificado a expressão do ciclo sono/vigília nos indivíduos por todo o mundo².

O ciclo sono/vigília é um ritmo circadiano que em condições naturais apresenta sincronização com fatores ambientais e varia no decorrer do período de 24 horas. Alguns fatores são sincronizadores deste ciclo, tais como alternância do claro-escuro (dia-noite), os horários de trabalho, horários de lazer, as atividades familiares e atividades sociais, sendo considerados fatores exógenos. Além dos fatores exógenos, o ciclo sono e vigília também é regulado por fatores endógenos, representados pela 
secreção de hormônios como o cortisol, a melatonina e a somatotropina (hormônio do crescimento) ${ }^{3}$.

O equilíbrio entre os fatores sincronizadores é fundamental para um sono de qualidade. Quando este equilíbrio é perturbado por mudanças abruptas em jornada de trabalho, voos trans-meridianos ou trabalho noturno ${ }^{4}$, pode desencadear uma série de alterações como transtorno do sono, transtornos de humor (irritabilidade, tensão, confusão, ansiedade), sensação de mal-estar, alterações gastrointestinais e reduções no desempenho de tarefas que requerem atenção e concentração ${ }^{5}$.

O desalinhamento circadiano provocado pela alteração sistemática de horas de sono, decorrentes de alterações comportamentais, sociais ou laborais é a principal razão para alguns distúrbios de sono ${ }^{6}$. A redução nas horas de sono dos indivíduos, tem influência do estresse, ingestão de medicamentos, obrigações sociais, irregularidade dos ciclos de sono-vigília e dos comportamentos de higiene do sono adotados?.

Dormir bem possibilita aos indivíduos a capacidade e prontidão para atender as demandas de trabalho. Por outro lado, os transtornos do sono podem ocasionar alterações significativas no funcionamento físico, cognitivo e ocupacional do indivíduo, além de comprometer consideravelmente a qualidade de vida3,8. Um sono insatisfatório e com baixa qualidade, pode trazer diversas repercussões para os seres humanos, como disfunção autonômica, aumento na incidência de transtornos 
psiquiátricos e metabólicos, com prejuízos diretos na saúde mental e consequentemente, no desempenho profissional do indivíduo ${ }^{9}$.

Algumas profissões podem trazer características estressantes. Ser professor pode ser uma dessas profissões. O trabalho docente é influenciado pelas transformações contemporâneas e a educação superior tornou-se uma alavanca para o desenvolvimento econômico do país e o conhecimento passou a ser visto por meio da produção científica desses trabalhadores. Nesse contexto, o processo de trabalho docente passou a demandar profissionais imediatistas, resolutivos e atentos a atualizações constantes. O meio científico passa a existir numa temporalidade de aceleração permanente em busca de produtividade $\mathrm{e}$ competência, em que se acentuam os dispositivos de controle que enfocam ligações funcionais e pragmáticas do processo de trabalho do professor em nível stricto sensu, tais como sistemas de avaliação e plataformas integradas de currículo.

Investigar a qualidade do sono e sua relação com a qualidade de vida e estado emocional, em professores universitários mostra-se necessário, uma vez que qualidade de sono ruim impacta diretamente na saúde física e emocional, podendo interferir, entre outros aspectos, no desempenho profissional, na saúde mental e na qualidade de vida. 
Neste contexto, o objetivo do estudo foi avaliar a qualidade do sono e sua relação com a qualidade de vida e estado emocional em professores universitários

\section{MÉTODO}

\section{Aspectos éticos}

A pesquisa teve início após aprovação do Comitê de Ética em Pesquisa do Centro Universitário de LavrasUnilavras (Parecer no 3.825.378). Trata-se de um estudo transversal seguindo as normas da Resolução 466/12 do Conselho Nacional de Saúde, relativa à pesquisa com seres humanos. Todos os participantes assinaram o Termo de Consentimento Livre e Esclarecido e/ou o Termo de Assentimento Livre e Esclarecido.

\section{Amostra}

Participaram do estudo 36 professores universitários de uma instituição de ensino superior privada no interior do estado de Minas Gerais.

Os critérios de elegibilidade foram todos os professores de ambos os gêneros, vinculados à instituição de ensino superior. Foram excluídos aqueles que não aceitaram participar da pesquisa, que não preencheram de forma adequada os questionários da pesquisa, professores com menos de 6 meses de vínculo empregatício com a Instituição e professores que estivessem afastados com licença laboral. 


\section{Procedimentos}

Inicialmente foi enviada uma mensagem através do email institucional para todo o corpo docente da instituição (132 professores), informando sobres os objetivos do estudo juntamente com um link (que dava acesso aos formulários online) caso eles optassem por participar voluntariamente da pesquisa. Dos 132 professores contactados, foram excluídos 96 (81 não responderam o e-mail, ou seja, não aceitaram participar da pesquisa e 15 foram excluídos por possuírem vínculo empregatício com a Instituição menor que 6 meses), totalizando então 36 voluntários.

A coleta de dados ocorreu no período de março a junho de 2020 através de um formulário eletrônico enviado no email de cada professor.

As variáveis sociodemográficas (gênero, idade, prática de atividade física, uso de medicamentos para dormir, ingestão de álcool para aliviar a tensão/ ansiedade) e as características de trabalho (tempo de trabalho na instituição, turnos trabalhados, demais vínculos empregatícios e carga horaria semanal trabalhada) foram avaliadas através de um questionário semiestruturado elaborado pelos autores do estudo.

Para avaliar a qualidade de sono foi utilizado o Índice de Qualidade de Sono de Pittsburgh (IQSP) que avalia a qualidade subjetiva do sono. Este questionário é composto por 19 itens agrupados em sete subescalas e a combinação das sete subescalas produz o escore global do IQSP que varia 
de 0 a 21. Escore final igual ou superior a 5 indica qualidade de sono ruim ${ }^{10}$.

Para avaliar a qualidade de vida foi utilizado o Questionário de Qualidade de Vida (SF-36). Este questionário consiste em duas partes; uma avalia o Componente Físico e outra avalia o Componente Mental, apresentando um escore final de 0 a 100, no qual zero corresponde ao pior estado geral de saúde e 100 ao melhor estado de saúde ${ }^{11}$.

Para a avaliação do estado emocional foi utilizado a Escala de Ansiedade, Depressão e Estresse EADS-21. Tratase de um instrumento de autorrelato constituído por 21 itens, divididos em três componentes (ansiedade, depressão e estresse). O escore final varia de 0 a 21 e quanto mais elevada a pontuação, mais negativos são os estados emocionais do indivíduo ${ }^{12,13}$. Os pontos de corte recomendados para cada subescala são os seguintes: Ansiedade [normal (0-7), leve (8-9), moderada (10-14), grave (15-19), muito grave (maior que 20)]; Depressão [normal (0-9), leve (10-13), moderada (14-20), grave (2127), muito grave (maior que 28)]; Estresse [normal (0-14), leve (15-18), moderada (19-25), grave (26-33), muito grave (maior que 34$)]^{14}$.

\section{Análise estatística}

Os dados foram analisados por meio de estatísticas descritivas, usando tabelas e gráficos de distribuição de frequência. As correlações entre qualidade do sono e saúde 
mental, qualidade do sono e qualidade de vida foram realizados através do coeficiente de correlação de Spearman. Foram utilizados os softwares Excel e o R 4.0.

\section{RESULTADOS}

\section{Caracterização da amostra}

Participaram da pesquisa 36 professores de graduação, de uma instituição privada, sendo 10 do gênero masculino $(27,8 \%)$ e 26 do gênero feminino $(72,2 \%)$, com média de idade de $38,61 \pm 7,75$.

\section{Dados sociodemográficos}

A maioria dos participantes eram casados $(58,3 \%)$, realizava atividade física de 3 a 4 vezes por semana $(44,4 \%)$, não utilizava medicamentos para dormir $(88,8 \%)$ e não fazia ingestão de álcool com o objetivo de aliviar a tensão $(94,4 \%)$.

\section{Características do trabalho}

Foi possível observar que a maioria dos participantes trabalhava na instituição há 5 anos ou menos $(47,2 \%)$, trabalhava dois turnos diários $(55,6 \%)$ e não possuía outros vínculos empregatícios (55,6\%). Em relação a carga horária semanal trabalhada, a maioria $(58,3 \%)$ trabalhava de 21 a 40 horas. 


\section{Qualidade do Sono em relação a Qualidade de Vida}

A prevalência de qualidade ruim do sono foi de $58,3 \%$. Ao avaliar a qualidade do sono em relação a qualidade de vida, foi possível observar que a maioria dos componentes da qualidade de vida possuem correlação com a qualidade do sono, com exceção dos componentes: estado geral de saúde e saúde mental (Tabela 1 ).

Tabela 1. Coeficiente de correlação $(\rho)$ de Spearman e valores p para o teste de correlação entre Qualidade do Sono e Qualidade de Vida.

\begin{tabular}{lccc}
\hline \multirow{2}{*}{ Qualidade de Vida } & \multicolumn{3}{c}{ Qualidade do Sono } \\
\cline { 2 - 4 } & $\mathrm{n}$ & $\begin{array}{c}\text { Coeficiente de Correlação } \\
\text { de Spearman }\end{array}$ & Valor $\mathrm{p}$ \\
\hline Capacidade Funcional & 36 & $-0,3623$ & $\mathbf{0 , 0 2 9 8 8}$ \\
Limitação por Aspecto Físico & 36 & $-0,3812$ & $\mathbf{0 , 0 2 1 8 1}$ \\
Dor & 36 & $-0,4668$ & $\mathbf{0 , 0 0 4 1 1}$ \\
Estado Geral de Saúde & 36 & $-0,1796$ & 0,29450 \\
Vitalidade & 36 & $-0,3874$ & $\mathbf{0 , 0 1 9 5 6}$ \\
Aspectos Sociais & 36 & $-0,5966$ & $\mathbf{0 , 0 0 0 1 2}$ \\
Limitação por Aspecto Emocional & 36 & $-0,3314$ & $\mathbf{0 , 0 4 8 3 4}$ \\
Saúde Mental & 36 & $-0,2839$ & $\mathbf{0 , 0 9 3 3 9}$ \\
\hline
\end{tabular}

\section{Qualidade do Sono em relação ao Estado Emocional}

75\% dos participantes não apresentaram sinais de ansiedade e depressão e $77,8 \%$ não apresentaram sinais de estresse.

Ao analisar a relação da qualidade do sono com o estado emocional, observa-se que somente a ansiedade apresentou correlação com a qualidade do sono ( $p=0,00078$; Tabela 2$)$. 
Tabela 2. Coeficiente de correlação $(\rho)$ de Spearman e valores p para o teste de correlação entre Qualidade do Sono e Saúde Mental.

\begin{tabular}{lccc}
\hline & \multicolumn{3}{c}{ Qualidade do Sono } \\
\cline { 2 - 4 } Saúde Mental & $\mathrm{N}$ & $\begin{array}{c}\text { Coeficiente de } \\
\text { Correlação de } \\
\text { Spearman }\end{array}$ & Valor p \\
\hline Ansiedade & 36 & 0,5346 & $\mathbf{0 , 0 0 0 7 8}$ \\
Depressão & 36 & 0,2798 & 0,09842 \\
Estresse & 36 & 0,3029 & 0,07258 \\
\hline
\end{tabular}

\section{DISCUSSÃO}

O objetivo do estudo foi avaliar a qualidade do sono e sua relação com a qualidade de vida e estado emocional de professores universitários.

A maioria $(58,3 \%)$ dos participantes apresentou qualidade de sono ruim. Essa frequência é semelhante a inúmeros estudos que avaliaram a qualidade do sono de professores universitários ${ }^{15}$. Infere-se que o processo de trabalho docente caracterizado pelas exigências de produtividade pode induzir os docentes a desenvolver muitos projetos concomitantes ou acelerar o ritmo de trabalho. A qualidade de sono ruim em professores universitários pode ser resultado de um ritmo de vida acelerado a que esses profissionais são submetidos. Professores universitários necessitam preparar e ministrar aulas, preparar, aplicar e corrigir provas, dar atenção extra a seus alunos, produzir artigos acadêmicos, elaborar trabalhos de pesquisas, orientar alunos, entre outras inúmeras atividades que 
aumentam a demanda de carga horária extra e muitas vezes interfere diretamente na vida pessoal e em especial no sono. Muitas vezes estes profissionais sacrificam o período que seria destinado para dormir realizando as tarefas extras que são exigidas pelo trabalho, tendo o costume de se abster-se de horas de sono que seriam essenciais para saúde mental e qualidade de vida ${ }^{16,17}$.

Quando avaliado o estado emocional dos professores através da EADS-21, foi encontrado que a maioria dos participantes não apresentavam sinais de ansiedade (75\%), depressão (75\%) e estresse (77,8\%). Porém quando avaliado a correlação entre qualidade do sono e os componentes do EADS-21 (ansiedade, depressão e estresse), foi encontrado correlação entre a qualidade do sono e ansiedade.

A literatura evidencia que a ansiedade tem sido apontada como uma das principais consequências da privação do sono, sobretudo na forma de transtorno de ansiedade generalizada ${ }^{18}$.

Aspectos sociais, como fatores de estilo de vida, o estresse e a ansiedade influenciam os padrões de sono ${ }^{19}$. Indivíduos com insônia exibem níveis mais altos de ansiedade e depressão ${ }^{20}$.

Foi observado em docentes de ensino superior uma alta prevalência de sintomas de insônia e de qualidade de sono ruim $^{21}$. A fadiga relacionada ao trabalho e o sedentarismo apresentaram-se como fatores precipitantes para desenvolvimento da insônia. A insônia tem impactos 
negativos na avaliação de domínios da qualidade de vida, além de se estenderem às relações sociais, saúde física e emocional ${ }^{22,23}$.

Os resultados do presente estudo mostraram correlação significativa entre qualidade do sono e a maioria dos componentes da qualidade de vida, exceto os componentes: estado geral de saúde e a saúde mental.

Já é consenso na literatura que a qualidade do sono está intimamente ligada a qualidade de vida do ser humano ${ }^{24,25}$, porém mais do que comprometer as noites de sono, dormir mal impacta na saúde física, cognitiva e emocional ${ }^{7,9,26}$. Dormir mal impacta negativamente o estado geral de saúde, afetando a capacidade funcional do indivíduo, o bem estar físico e psicológico, a vitalidade, os aspectos sociais, entre outros, evidenciando que alterações no sono podem atrapalhar a qualidade de vida25,27,28. Esses hábitos podem ter consequências a curto e a longo prazo na saúde e segurança dos trabalhadores, pois ocasionam queda das funções cognitivas, no raciocínio e no julgamento, tornandoos mais propensos a erros e prejudicando o processo de trabalho docente que envolve majoritariamente aspectos cognitivos.

Caso as condições para uma melhor qualidade de vida e uma noite boa de sono não sejam adquiridas, podem aparecer danos à sociedade, já que os professores são a base de formação, desde o conhecimento em nível básico até o que há de mais atualizado e avançado nas pesquisas em diversas áreas ${ }^{29}$. 
É importante que a sociedade se atente com os contextos de saúde, em particular as relacionadas ao sono, para que, assim, possa prosperar e desenvolver-se de forma concreta e estruturada. As implicações se estendem da docência para todos os domínios trabalhistas e funcionais, já que, a cada novo estudo, se verifica que os melhoramentos acrescidos de uma vida repleta de práticas saudáveis são maiores do que um projeto de reestruturação ou uma inovação na forma de abordagem dos desafios diários ${ }^{30}$.

Vale ressaltar que os resultados desta pesquisa apresentam algumas limitações: a amostra foi coletada de uma única universidade, portanto a generalização dos resultados deve ser feita cautelosamente e o sono foi avaliado em uma única aplicação, impedindo de obter resultados mais detalhados. Pesquisas futuras podem procurar determinar se os resultados do estudo são aplicáveis em outros professores.

Todavia, o presente estudo contribui com a discussão da temática no que tange à influência da qualidade do sono na qualidade de vida e saúde emocional de professores universitários. Como implicações práticas, dadas as oportunidades limitadas de influenciar aspectos pessoais dos trabalhadores, uma alternativa aos gestores é estimular um ambiente de trabalho em que sejam adotadas políticas e práticas de pessoal que limitem os docentes a trabalhar excessivamente em nível estrutural ou fornecer recursos para equilibrar as demandas de trabalho. 


\section{CONCLUSÃO}

Os professores avaliados no presente estudo apresentaram em sua maioria qualidade do sono ruim. Aqueles que apresentam qualidade do sono ruim, apresentaram também sinais significantes de ansiedade e má qualidade de vida, ou seja, a qualidade do sono está relacionada a qualidade de vida e estado emocional (ansiedade).

\section{REFERÊNCIAS}

1.Bonmati-Carrion MA, Middleton B, Revell V, Skene DJ, Rol MA, Madrid JA. Circadian phase asessment by ambulatory monitoring in humans: Correlation with dim light melatonin onset. Chronobiol Int 2014;31:3751. https://doi.org/10.3109/07420528.2013.820740

2. Bin YS, Marshall NS, Glozier N. Secular trends in adult sleep duration: A systematic review. Sleep Med Rev 2012;16:223-30. http://dx.doi.org/10.1016/j.smrv.2011.07.003

3. Marques DR, Meia-Via AMS, Fernandes C, Gomes AA. Associations between sleep quality and domains of quality of life in a non-clinical sample: results from higher education students. Sleep Heal J Natl Sleep Found 2017;3:348-56. http://dx.doi.org/10.1016/j.sleh.2017.07.004 4. Monk TH, Buysse DJ, Billy BD, Kennedy KS, Willrich LM. Sleep and Circadian Rhythms in Four Orbiting Astronauts. J Biol Rhythms 1998;13:188-201. https://doi.org/10.1177/074873098129000039

5. Gaspar S, Moreno C, Menna-Barreto L. Os plantões médicos, o sono e a ritmicidade biológica. Rev Assoc Med Bras 2005;44:239-45. https://doi.org/10.1590/s0104-42301998000300013

6.Garbarino S, Magnavita N. Obstructive Sleep Apnea Syndrome (OSAS), Metabolic Syndrome and Mental Health in Small Enterprise Workers. Feasibility of an Action for Health. PLoS One 2014;9:5-10. https://doi.org/10.1371/journal.pone.0097188

7.Lawson HJ, Wellens-mensah JT, Nantogma SA. Evaluation of Sleep Patterns and Self-Reported Academic Performance among Medical Students at the University of Ghana School of Medicine and Dentistry. Sleep Disord 2019;2019:1-9. https://doi.org/10.1155/2019/1278579 8.Ge Y, Xin S, Luan D, Zou Z, Liu M, Bai X, et al. Association of physical activity, sedentary time, and sleep duration on the health-related quality of life of college students in Northeast China. Helth Qual life outcomes 2019;17:1-8. https://doi.org/10.1186/s12955-019-1194-X 9. Orzeł-Gryglewska J. Consequences of sleep deprivation. Int J Occup Med Environ Health 2010;23:95-114. https://doi.org/10.2478/v10001-010-0004-9 
10.Buysse DJ, Reynolds CF, Monk TH, Berman SR, Kupfer DJ. The Pittsburgh Sleep Quality Index: a new instrument for psychiatric practice and research. Psychiatry Res 1989;28:193-213. https://doi.org/10.1016/0165-1781(89)90047-4

11.Vitorino DFM, Martins FLM, Souza AC, Prado GF. Utilização do SF36 em ensaios clínicos envolvendo pacientes fibromiálgicos. Rev Neurocienc

https://doi.org/10.4181/rnc.2004.12.147

2004;12:147-51.

12.Vignola RCB, Tucci AM. Adaptation and validation of the depression, anxiety and stress scale (DASS) to Brazilian Portuguese. J Affect Disord 2014;155:104-9. http://dx.doi.org/10.1016/j.jad.2013.10.031

13.Pais-Ribeiro JL, Honrado A, Leal I. Contribuição para o estudo da adaptação portuguesa das escalas de ansiedade, depressão e stress (EADS) de 21 itens de lovibond e lovibond. Psicol Saúde Doenças 2004;5:229-39.

aberto.up.pt/bitstream/10216/6910/2/81876.pdf

https://repositorio-

14.Antony MM, Cox BJ, Enns MW, Bieling PJ, Swinson RP. Psychometric properties of the 42-item and 21-item versions of the Depression Anxiety Stress Scales in clinical groups and a community sample. Psychol Assess 1998;10:176-81. https://doi.org/10.1037/10403590.10.2.176

15.Cardoso MG, Mesas AE, Cardelli AAM, Galdino JM, Barreto MFC, Aroni $P$, et al. Qualidade do sono e workaholism em docentes de pósgraduação stircto sensu. Acta Paul Enferm 2020;33:1-8. https://doi.org/10.37689/acta-ape/2020A002285

16.Amaro JMRS, Dumith SC. Excessive daytime sleepiness and quality of life related to the health of university professors. J Bras Psiquiatr 2018;67:94-100. https://doi.org/10.1590/0047-2085000000191

17. de Oliveira ERA, Garcia ÁL, Gomes MJ, Bittar TO, Pereira AC. Gênero e qualidade de vida percebida-Estudo com professores da área de saúde. Cienc Saude Col 2012;17:741-7. https://doi.org/10.1590/S1413-81232012000300021

18. Leite BR, Vieira TFS, Mota ML, Nascimento EC, Gomes ICP. Associação entre qualidade do sono e ansiedade em acadêmicos de medicina. Braz J Heal Rev 2020;3:6528-43. https://doi.org/10.34119/bjhrv3n2-075

19.Almojali AI, Almalki SA, Alothman AS, Masuadi EM, Alaqeel MK. The prevalence and association of stress with sleep quality among medical students. J Epidemiol Glob Health 2017;7:169-74. http://dx.doi.org/10.1016/j.jegh.2017.04.005

20.Patrick Y, Lee A, Raha O, Pillai K, Gupta S, Sethi S, et al. Effects of sleep deprivation on cognitive and physical performance in university students. Sleep Biol Rhythms 2017;15:217-25. https://doi.org/10.1007/s41105-017-0099-5

21.Schattan RB. Saúde, ambiente e mudanças sociais, fatores associados ao sono de docentes universitários (Tese). Universidade Católica de Santos, 2017, 171 p.

22.Bos SC, Macedo AF. Literature review on Insomnia (2010-2016). Biol

Rhythm

Res

2019;50:94-163. 
http://doi.org/10.1080/09291016.2017.1413766

23. Guimarães SS, Muller MR. Impacto dos transtornos do sono sobre o funcionamento diário e a qualidade de vida 1 Sleep disorders impact on daily functioning. Estud Psicol Campinas 2007;24:519-28. https://doi.org/10.1590/S0103-166X2007000400011

24.Barros MA, Lima MG, Ceolim MF, Zancanella E, Cardoso TAMO. Qualidade do sono, saúde e bem-estar em estudo de base populacional. Rev Saude Pub 2019;53:1-12. http://doi.org/10.11606/s1518-8787.2019053001067

25. Hoefelmann LP, Lopes S, Samara K, Ginar S, Gatto L, Cabral A, et al. Lifestyle, self-reported morbidities, and poor sleep quality among Brazilian workers. Sleep Med 2012;13:1198-201. http://dx.doi.org/10.1016/j.sleep.2012.05.009

26.Weber F. ScienceDirect Modeling the mammalian sleep cycle. Curr Opin Neurobiol

http://dx.doi.org/10.1016/j.conb.2017.07.009 2017;46:68-75.

27.Rezaei M, Khormali M, Akbarpour S, Sadeghniiat-Hagighi K, Shamsipour M. Sleep quality and its association with psychological distress and sleep hygiene: a cross-sectional study among pre-clinical medical students. Sleep Sci 2018;11:274-80.

http://doi.org/10.5935/1984-0063.20180043

28.Chattu VK, Manzar D, Kumary S, Burman D, Spence DW, Pandiperumal SR. The global problem of insufficient sleep and its serious public health implications. Healtcare 2019;7:1-16. http://doi.org/10.3390/healthcare7010001

29. Crepaldi TOM, Carvalhais JDJ. A contribuição da má qualidade do sono na qualidade de vida no trabalho de professores: Uma revisão. Brazilian J Dev 2020;6:75044-57. http://doi.org/10.34117/bjdv6n10070

30.Koetz L, Rempel C, Périco E. Qualidade de vida de professores de Instituições de Ensino Superior Comunitárias do Rio Grande do Sul. Cien Saude Colet 2013;18:1019-28. http://doi.org/10.1590/s141381232013000400015 